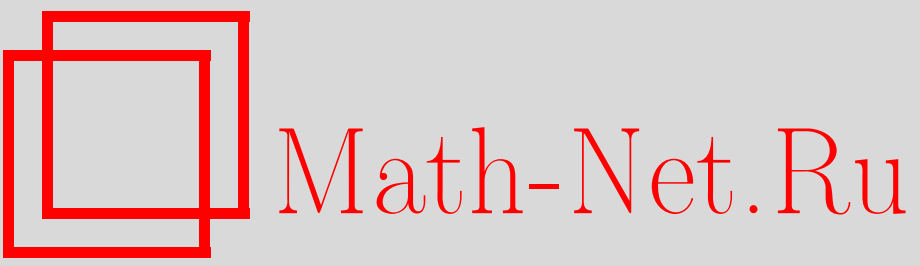

С. А. Богатый, Г. С. Скордев, Теорема совпадения для $M$-подобных континуумов, УМН, 2002, том 57, выпуск 2, 189-190

DOI: https://doi.org/10.4213/rm502

Использование Общероссийского математического портала Math-Net.Ru подразумевает, что вы прочитали и согласны с пользовательским соглашением

http://www.mathnet.ru/rus/agreement

Параметры загрузки:

IP: 54.209 .52 .79

26 апреля 2023 г., 14:04:18 


\title{
ТЕОРЕМА СОВПАДЕНИЯ ДЛЯ $M$-ПОДОБНЫХ КОНТИНУУМОВ
}

\author{
С. А. БОГАТЫЙ, Г. С. СКОР ДЕВ
}

При исследовании динамических свойств рационального (или даже полиномиалнного) отображения $f: S^{2} \rightarrow S^{2}$ сферы Римана в себя бывает полезно рассмотреть ламинацию $\left(S^{2}, f\right)$. В работе исследуются свойства ламинации, близкие к свойству неподвижной точки. При этом мы рассматриваем ламинации, полученные "переменньпм" отображениями фиксированного связного компактного ориентированного многообразия $M^{n}$ без края. Известно [1; теорема $\left.1^{*}\right]$, что компактное пространство $Y$ представимо в виде предела обратной последовательности

$$
M_{1}^{n} \stackrel{p_{1}^{2}}{\longleftarrow} M_{2}^{n} \longleftarrow p_{2}^{3}
$$

$n$-мерных многообразий без края тогда и только тогда, когда для любого $\varepsilon>0$ существует $\varepsilon$-отображение $f_{\varepsilon}: Y \rightarrow M^{n}$.

Мы будем предполагать, что в спектрах, задающих компакты $X$ и $Z$, все связьвающие отображения имеют ненулевую степень. Про спектр, задающий компакт $Y$, будем дополнительно предполагать, что все пространства гомеоморфны фиксированному многообразию $M$.

Всякое отображение $f: M \rightarrow M$ многообразия в себя ненулевой степени (например, полиномиальное или рациональное отображение сфферы $M=S^{2}$ ) порождает такое пространство $Y=(M, f)$ - обратный предел последовательности $\mathscr{M}$, в которой все связывающие отображения $p_{i}^{i+1}$ совпадают с $f$. Согласно теоремам Понтрягина и Баума [2; следствие 3] всякая связная компактная топологическая группа $G$ конечной размерности $n$ представима в виде обратного предела последовательности $\mathscr{M}$, в которой пространство $M$ является связной компактной группой Ли, а связывающие отображения $p_{i}^{i+1}$ являются гомоморфизмами, в частности, накрытиями, поэтому имеют ненулевую степень.

Пусть $X$ и $Z$ являются пределами спектров $\mathcal{N}$ и $\mathscr{M}$ соответственно из $n$-мерных многообразий без края со связывающими отображениями $\pi_{i}^{j}$ и $p_{i}^{j}$ ненулевой степени и предельными проекциями $\pi_{i}: X \rightarrow N_{i}^{n}$ и $p_{i}: Z \rightarrow M_{i}^{n}$ соответственно. Известно, что для отображения $f: X \rightarrow Z$ для всякого $\varepsilon>0$ и всякого индекса $i$ существуют такой индекс $\varphi(i)$ и такое отображение $f_{i}: N_{\varphi(i)} \rightarrow$ $M_{i}$, что $\varrho\left(f_{i} \circ \pi_{\varphi(i)}, p_{i} \circ f\right)<\varepsilon$.

Cтепенью $\operatorname{deg}_{\mathcal{N}, \mathscr{M}} f$ отображения $f$ назьвается число

$$
\operatorname{deg}_{\mathscr{N}, \mathscr{M}}(f)=\operatorname{deg}\left(f_{i}\right) \cdot \operatorname{deg} p_{1}^{i} / \operatorname{deg} \pi_{1}^{\varphi(i)} .
$$

Если число $\varepsilon$ настолько мало, что любые два $\varepsilon$-близкие отображения гомотопны, то определенное нами число не зависит от взятой аппроксимации (в частности, от индекса $i$ ). Доказательство легко вытекает из гомотопической инвариантности и мультипликативности степени для отображений многообразий. Степень обладает следующими важными свойствами:

(1) $\operatorname{deg} \mathscr{M}, \mathscr{M}(\mathrm{Id})=1$;

(2) $\operatorname{deg}_{\mathscr{K}, \mathscr{M}}(f \circ g)=\operatorname{deg}_{\mathscr{K}, \mathcal{N}}(g) \cdot \operatorname{deg} \mathscr{N}, \mathscr{M}(f)$.

В случае, когда все многообразия $M_{i}$ гомеоморфны, можно корректно определить число Лефьшеца (число совпадения). Для пары отображений $f, g: X \rightarrow Y$ рассмотрим индекс, допускающий одновременную аппроксимацию $f_{i}, g_{i}: N_{\varphi(i)}^{n} \rightarrow M_{i}^{n}$. Положим $\Lambda_{\mathcal{N}}, \mathscr{M}(f, g)=\Lambda\left(f_{i}, g_{i}\right)$. $\operatorname{deg} p_{1}^{i} / \operatorname{deg} \pi_{1}^{\varphi(i)}$, где $\Lambda\left(f_{i}, g_{i}\right)$ - обычное число Леффшеца отображения многообразий. Корректность введенного числа легко вытекает из следующего предложения, которое в свою очередь вытекает из теоремы Хопфа [3; теорема 14.2.5].

ПредлОЖение 1. Если $\pi: K^{n} \rightarrow N^{n}$ u $p: M^{n} \rightarrow M^{n}$ - отображсения ненулевой степени, то для всякой пары отображений $f, g: N^{n} \rightarrow M^{n}$ справедливо равенство:

$$
\Lambda(p \circ f \circ \pi, p \circ g \circ \pi)=\operatorname{deg}(\pi) \cdot \operatorname{deg}(p) \cdot \Lambda(f, g) .
$$

Работа выполнена во время визита первого автора в университет г. Бремен при частичной поддержке РФФИ (грант № 00-01-00304). Авторы благодарят Х.-О. Пайтгена и Х. Цишанга за поддержку. 
Следовательно, $\Lambda\left(f_{i}, g_{i}\right) \cdot \operatorname{deg} p_{1}^{i} / \operatorname{deg} \pi_{1}^{\varphi(i)}=\Lambda\left(p_{1}^{i} \circ f_{i}, p_{1}^{i} \circ g_{i}\right) / \operatorname{deg} \pi_{1}^{\varphi(i)}=\Lambda\left(f_{1} \circ \pi_{\varphi(1)}^{\varphi(i)}\right.$, $\left.g_{1} \circ \pi_{\varphi(1)}^{\varphi(i)}\right) / \operatorname{deg} \pi_{1}^{\varphi(i)}=\Lambda\left(f_{1}, g_{1}\right) / \operatorname{deg} \pi_{1}^{\varphi(1)}$. В доказательстве мы считали, что индекс $\varphi(i)$ так велик, что отображения $p_{1}^{i} \circ f_{i}$ и $f_{1} \circ \pi_{\varphi(1)}^{\varphi(i)}\left(\right.$ соответственно $p_{1}^{i} \circ g_{i}$ и $\left.g_{1} \circ \pi_{\varphi(1)}^{\varphi(i)}\right)$ гомотопны.

Теорема 1. Если для двух отображсений $f, g: X \rightarrow Y$ число совпадения $\Lambda_{\mathcal{N}, \mathscr{M}}(f, g)$ $\neq 0$, то существует такая точка $x \in X$, что $f(x)=g(x)$.

ДокАЗАТЕЛЬСТвО. Если $f(x) \neq g(x)$ для всякой точки $x \in X$, то существует такое число $\varepsilon>0$, что $\varrho(f(x), g(x)) \geqslant 4 \varepsilon$ для всех $x \in X$. Тогда существует такой индекс $i$, что $\varrho\left(p_{i} \circ f(x)\right.$, $\left.p_{i} \circ g(x)\right) \geqslant 3 \varepsilon$ для всех $x \in X$. Следовательно, для некоторого индекса $\varphi(i) \varrho\left(f_{i} \circ \pi_{\varphi(i)}(x)\right.$, $\left.g_{i} \circ \pi_{\varphi(i)}(x)\right) \geqslant \varepsilon$ для всех $x \in X$. Но $\Lambda\left(f_{i}, g_{i}\right)=\Lambda_{\mathcal{N}, \mathscr{M}}(f, g) \cdot \operatorname{deg} \pi_{1}^{\varphi(i)} / \operatorname{deg} p_{1}^{i} \neq 0$, поэтому согласно классической теореме Лефшеца существует такая точка $\widetilde{x} \in X_{\varphi(i)}$, что $f_{i}(\widetilde{x})=g_{i}(\widetilde{x})$.

Как и в случае отображения многообразий, если $c: X \rightarrow Y$-постоянное отображение в одну точку, то $\Lambda_{\mathcal{N}, \mathscr{M}}(f, c)=\operatorname{deg}_{\mathscr{N}}, \mathscr{M}(f)$. Хотя введенное число совпадения зависит от представляющих спектров, можно получить некоторые "абсолютные" соотношения. Например, справедлив аналог теоремы Фаделла-Брукса-Вонга [4; теорема 3.22].

ТеОРема 2. Если $Y=G$ является связной компактной $n$-мерной группой, то для всякой пары отображений $f, g: X \rightarrow G$ справедливо равенство:

$$
\Lambda_{\mathcal{N}, \mathscr{M}}(f, g)=\operatorname{deg}_{\mathscr{N}, \mathscr{M}}(f / g) .
$$

ПредЛОженИЕ 2. Если $\pi: Z \rightarrow X u p: Y \rightarrow Y$ - отображения ненулевой степени, то для всякой пары отображений $f, g: X \rightarrow Y$ справедливо равенство:

$$
\Lambda_{\mathscr{K}, \widetilde{\mathscr{M}}}(p \circ f \circ \pi, p \circ g \circ \pi)=\operatorname{deg}_{\mathscr{K}, \mathcal{N}}(\pi) \cdot \operatorname{deg}_{\mathscr{M}, \widetilde{M}}(p) \cdot \Lambda_{\mathcal{N}, \mathscr{M}}(f, g) .
$$

Фактически с помощью спектров $\mathscr{N}$ и $\mathscr{M}$ в пространствах $X$ и $Y$ введена двойственность Пуанкаре и далее число совпадения определяется стандартным образом как алштернированная сумма следов индуцированных гомоморфизмов гомологий и когомологий Чеха с рациональными коэффициентами. Степень отображения также определяется индуцированным гомоморфизмом в старших гомологиях и когомологиях, но уже не имеет геометрической интерпретации как кратность. Рассмотрим отображение единичной окружности $f: S^{1} \rightarrow S^{1}$, переводящее точку в ее квадрат. Тогда обратньй предел $\left(S^{1}, f\right)$ является 2-адическим соленоидом. Степень индуцированного гомеоморфизма сдвига $\widehat{f}:\left(S^{1}, f\right) \rightarrow\left(S^{1}, f\right)$ равна 2 .

В случае $X=Y$ и $g=\mathrm{Id}$ число $\Lambda_{\mathscr{M}, \mathscr{M}}(f, \mathrm{Id})$ можно определить по схеме Хопфа, пользуясь только гомологиями или когомологиями с рациональньми коэффициентами. Для связной компактной конечномерной группы три задачи нахождения совпадения, неподвижной точки и корня эквивалентны.

СлЕДСтВиЕ. Если $X=Y=G$ является связной компактной $n$-мерной группой, то для всякой пары отображений $f, g: G \rightarrow G$ справедливо равенство:

$$
\Lambda_{\mathscr{M}, \mathscr{M}}(f, g)=\Lambda_{\mathscr{M}, \mathscr{M}}(h, \mathrm{Id})=\operatorname{deg}_{\mathscr{M}, \mathscr{M}}(f / g),
$$

где $h(x)=f(x) \cdot x / g(x)$

\section{СПИСОК ЛИТЕРАТУРЫ}

[1] S. Mardešić, J. Segal // Trans. Amer. Math. Soc. 1963. V. 109. №1. P. 146-164. [2] P. F. Baum // Pacific J. Math. 1967. V. 22. № 2. P. 197-204. [3] R. Stöcker, H. Zieschang. Algebraische Topologie. Stuttgart: Teubner, 1988. [4] С. А. Богатый, Д. Л. Гонсалвес, Х. Цишанг // Труды МИАН. 1999. Т. 225. С. 52-86.

Московский государственньй университет им. М. В. Ломоносова; Факултет математики университета Бремена, Германия 